\title{
ARTÍCULOS
}

CRÍTICA, Revista Hispanoamericana de Filosofía

Vol. XXXII, No. 95 (agosto 2000): 3-46

\section{UNA TEORÍA COMBINATORIA DE LAS REPRESENTACIONES CIENTÍFICAS}

Andoni IBARRA Unidad de Filosofía de la Ciencia Universidad del País Vasco/CSIC

Thomas Mormann

Departamento de Lógica y Filosofía de la Ciencia Universidad del País Vasco

1. Introducción

2. De la isomorfía a la homología

3. Aspectos combinatorios de las representaciones científicas

4. El grupo fundamental de Poincaré

5. Representaciones homológicas en la ciencia empírica

6. La ciencia como representación

Referencias bibliográficas

\section{Introducción}

Desde hace ya algún tiempo el concepto de representación se ha convertido en blanco de ataque de diversas posiciones filosóficas. Rorty y otros neopragmatistas consideran que ese concepto conduce inevitablemente a un laberinto de callejones sin salida y pseudoproblemas irresolubles. En este artículo nos proponemos mostrar que la noción de representación debe desempeñar una función central en la filosofía y, en particular, en la filosofía de la ciencia. Por 
supuesto, no cualquier noción de representación está capacitada para ese empeño. Rorty, por ejemplo, tiene razón cuando afirma que su noción de representación carece de utilidad. La representación, para él, equivale a reflejo especular (Rorty 1979, p. 12). Este enfoque de la representación como reflejo viene caracterizado por la idea de que lo representado y lo representante son en gran parte semejantes; lo son, en concreto, en la medida en que uno es la imagen especular del otro. Este tipo de representación no desempeña efectivamente ninguna función ni en la ciencia ni en la filosofía. La representación como reflejo especular o, expresado en términos matemáticos, la representación como isomorfismo, es un caso especial poco relevante de la representación. La representación realmente existente, esto es, la que podemos observar en la práctica efectiva de la ciencia, es un concepto complejo y "difícil" que requiere de elaboración y elucidación: en otras palabras, es un concepto que para su comprensión necesita una teoría de las representaciones científicas.

En este artículo introducimos un nuevo concepto general de representación. La tesis central es que nuestro concepto de representación capta las propiedades esenciales de las representaciones científicas mejor que otros conceptos ya propuestos en la literatura. El nuevo concepto de representación, que bautizaremos como representación homológica o functorial por motivos que se harán obvios más adelante, es una generalización del concepto de representación concebido como aplicación (parcialmente) preservadora de estructura, tal como ha sido elucidado por diversos autores (por ejemplo, Mundy 1986, Swoyer 1989, Krantz et al. 1971-1990).

Por supuesto, aquí no podemos ofrecer un enfoque comprehensivo de la naturaleza de la representación functorial y su papel en la práctica científica. Por lo tanto, nos conformaremos con explicar los lineamientos básicos de ese 
enfoque de manera razonablemente detallada y explorar su aplicabilidad en un par de ejemplos. Las tesis centrales que serán desarrolladas a lo largo del trabajo son, esquemáticamente expresadas, las siguientes:

(1) La representación no es un reflejo especular; ésta o, más precisamente, las representaciones isomórficas carecen de interés. La representación es un concepto complejo, que requiere de una teoría para su elucidación. Una de las tareas capitales de una buena teoría de las representaciones científicas es justamente la de armar de manera razonable el carácter no-isomórfico de la representación científica. En el presente trabajo se expone en este sentido el concepto de representación homológica, el concepto más "no-isomórfico" de los propuestos hasta ahora en la literatura concerniente a la representación.

(2) La matemática constituye un importante soporte de las representaciones científicas, pero no el único: existen también otros tipos de representaciones materiales que desempeñan una función esencial en esas representaciones. Una teoría general de las representaciones científicas debe procurar considerar todos los tipos de representaciones producidos en las prácticas de las ciencias.

(3) Las representaciones no aparecen aisladamente, sino en grupos $\mathrm{o}$, mejor, en sistemas. Las representaciones pueden combinarse e iterarse de diversos modos. Una teoría de la representación científica debe considerar esas posibilidades. O, expresándolo de manera más general, una teoría completa de las representaciones científicas debe dar cuenta del carácter híbrido de muchas representaciones. En la ciencia real las representaciones son con frecuencia sistemas mixtos de representaciones que combinan representaciones matemáticas con representaciones materiales de distintos tipos. 
(4) Las representaciones no son evidentes, no "hablan por sí mismas", necesitan ser interpretadas. Una buena parte de la práctica científica consiste en interpretar y reinterpretar representaciones. La representación es un concepto reflexivo y, consiguientemente, una teoría de las representaciones científicas debe considerar diversos tipos de representaciones de representaciones. Es decir, una teoría así puede concebirse como una teoría reflexiva combinatoria de las representaciones.

(5) El objetivo básico de las representaciones científicas es el razonamiento subrogatorio o razonamiento homológico. Este razonamiento permite transferir inferencias y resultados obtenidos en el dominio representante a propiedades y relaciones identificadas en el dominio representado. Como la estructura de aquél es más rica que la del dominio representado, el razonamiento subrogatorio permite explotar el rendimiento de las teorías identificables en el dominio representante aplicándolas al dominio representado. Una teoría de las representaciones científicas debe dar cuenta de este objetivo esencial de la práctica representacional.

Nuestro objetivo en este trabajo es contribuir a la construcción de esa teoría de las representaciones. El artículo está estructurado de este modo: en el apartado 2 presentamos el nuevo concepto básico de representación homológica, a partir de los enfoques más comunes de la representación, tales como la representación como sustitución o como aplicación (parcialmente) preservadora de estructura. En el apartado 3 esbozamos los lineamientos básicos de una teoría combinatoria de las representaciones, homológicas u otras. En el apartado 4 tratamos el ejemplo del grupo fundamental de Poincaré como un caso típico de representación homológica. En el apartado 5 consideramos algunos aspectos de las representaciones homológicas detectables en 
el proceso de teorización seguido en el siglo XX en el campo de las partículas subatómicas. Finalmente, en el apartado 6, describimos la práctica científica como una práctica representacional, esto es, como una actividad que consiste en la construcción, combinación y procesamiento de representaciones, tareas todas ellas que se desarrollan de manera diversa y plural.

\section{De la isomorfía a la homología}

El concepto de representación es un concepto con una amplia tradición filosófica. Aquí no podemos detenernos en los detalles de las discusiones existentes en esa tradición. Sírvanos en el presente contexto, y sin mayor justificación, tomar como punto de partida el concepto de representación propuesto por Peirce (Peirce 1973):

Una representación es siempre una representación de algo (A) por algo (B) para algo (C).

En primer lugar, una representación lo es de algo por algo. Denotamos una representación entendida en este sentido por $A \stackrel{r}{\longrightarrow} B$ o de manera similar. La relación de representación $r$ puede ser de diverso tipo. Si $A \stackrel{r}{\longrightarrow} B$ y $C \stackrel{r^{*}}{\longrightarrow} D$ designan conexiones representacionales, ello no implica que ambas conexiones sean semejantes. En general, la naturaleza de la relación de representación puede ser muy diferente en las distintas conexiones representacionales. No se presupone concretamente que $r$ sea una aplicación teórico-conjuntista o de naturaleza similar. La representación funciona en nuestra teoría general como un concepto primitivo, que puede ser interpretado de manera diferente en contextos distintos, y que en lo esencial se caracteriza por sus propiedades combinatorias relacionales —en el sentido que se precisará. 
Pero además la representación lo es para algo. Mientras que el papel de los ámbitos representado (A) y representante (B) es objeto de un análisis conspicuo en los trabajos de un buen número de estudiosos de la representación, el componente $(\mathrm{C})$ de nuestra definición de partida es apenas tratado, cuando no completamente ignorado, en muchos de esos trabajos. En el presente trabajo interpretamos (C) como el "contexto" o la "situación" representacional en la que se da una representación. Una representación es siempre una representación situada en un sentido fuerte, que procuraremos precisar.

En sus trabajos fundacionales sobre la semiótica general Peirce destacó persistentemente la significación central del "interpretante" (C) en toda conexión representacional o sígnica. Aun así, la evocación del concepto de interpretante por Peirce parece apuntar más a la identificación de un problema que a la formulación de su solución. En este sentido, la concepción peirceana del interpretante, en tanto que elemento de una semiótica general, ofrece tan sólo una contribución de carácter general para una teoría de la representación científica. Nuestro interés objetivo apunta a las características específicas de las representaciones científicas. Consiguientemente, en el apartado 3 procuramos explicitar el componente $(\mathrm{C})$ de la representación, mediante una teoría combinatoria de las representaciones científicas, en la cual el contexto realizador de la representación desempeña una función central, esto es, mediante una teoría en la que la representación científica capta su sentido y significación sólo en el contexto de una red realizadora de otras representaciones. Esta condición contextual o situada es aplicable a todo tipo de representaciones. Así pues, una teoría de la representación científica que tome en consideración los aspectos (A), (B) y (C) es en lo esencial una teoría combinatoria. 
Para empezar distinguimos cuatro tipos de representación identificables en las prácticas representacionales de la ciencia:

(1) la representación como isomorfía

(2) la representación como sustitución

(3) la representación como homomorfía

(4) la representación como homología

Dos acotaciones a esta clasificación. En primer lugar, ella no debe inducir la idea de que esas formas puras se hallen "realmente" en la práctica. Es una distinción operada por razones esencialmente metodológicas. En segundo lugar, la representación como isomorfía se menciona solamente por mor de la completud; de hecho, no desempeña ningún papel relevante en la práctica científica.

(1) La representación como isomorfía

Un enfoque intuitivo de la representación sostiene, en primer término, el requerimiento de la necesaria existencia de una semejanza entre los dos relatos de la representación. En las versiones más burdas del enfoque, la semejanza se identifica con la imagen especular (Rorty 1979). Las más refinadas constatan, sin embargo, la diversidad representacional y su irreductibilidad a la imagen anterior; aun así, postulan la identificabilidad de semejanzas — de tipo estructural o formal respecto de la organización relacional involucrada - entre el dominio representante y el representado. Un mecanismo arquetípico favorito de este enfoque es el descrito por la teoría fisiológica de la percepción cartesiana, según la cual se establece un isomorfismo completo entre los cuerpos externos, las vibraciones en la glándula pineal, las huellas en el cerebro y las sensaciones e ideas producidas. Las teorías neurofisiológicas actuales no requieren, sin embargo, que las transformaciones impresas en los órganos sensoriales, y transmitidas a través del 
sistema nervioso al cerebro, preserven isomórficamente la estructura del objeto.

En el dominio de la teorización más desarrollada, la geometría analítica de Descartes ha sido propuesta también como un caso relevante de construcción matemática sustentada en la idea de la representación isomórfica que aplica las entidades de la geometría a las entidades del álgebra (Suppes 1989). Recientemente algunos autores han pretendido ofrecer apoyo histórico a la idea de que la representación depende esencialmente de la semejanza isomórfica (Watson 1995). Una tesis colateral de nuestro trabajo es hacer convincente el argumento de la irrelevancia lógica y filosófica de la isomorfía para la representación científica.

(2) La representación como sustitución

Exceptuando la forma anterior de representación, la especie de representación más sencilla es la de sustitución. Se pueden encontrar ejemplos de esta especie en todos los dominios: el embajador de un país representa a ese país en una conferencia, un abogado representa a su cliente en un juicio, los padres de un menor actúan como apoderados de éste, un electo representa a "su" circunscripción electoral o a toda la ciudadanía de un país a través de su parlamento. Los números y otras magnitudes matemáticas funcionan como sustitutos vicariales de entidades empíricas de diverso tipo. Estas relaciones de representación por sustitución son distintas en muchos sentidos, y lo son además de manera muy diversa. Por ejemplo, la relación de representación entre un abogado y su cliente es de un tipo distinto al de la relación entre un electo y su circunscripción electoral.

(3) La representación como homomorfía

El interés prevaleciente en relación con la representación corresponde a la concepción de la representación como aplicación preservadora de estructura. La teoría representacional de la medida proporciona ejemplos típicos de esta clase 
de representaciones: por un lado, tenemos un dominio D de objetos empíricos a medir, y por el otro, en el lado representante, un dominio matemático, concretamente, el dominio $\mathbb{R}$ de los números reales (cfr. Krantz et al. 19711990, Narens 1985).

La medida como aplicación (parcialmente) preservadora de estructura consiste en hacer corresponder un número a cada objeto a medir, de tal manera que las relaciones empíricas como la longitud, la masa o similares se representen por relaciones numéricas. Este tipo de representación podemos describirlo formalmente como una aplicación $r$ : $D \longrightarrow \mathbb{R}$, tal que $r$ representa una operación $\otimes$ de concatenación empírica mediante la operación numérica de la adición:

$$
r(x \otimes y)=r(x)+r(y)
$$

Esta teoría de la representación concebida como una teoría de las aplicaciones preservadoras de estructuras se puede desarrollar, por lo tanto, en general, en el marco de una teoría de tales aplicaciones $r:\left(A, R_{1}, \ldots, R_{\mathrm{m}}\right) \longrightarrow$ $\left(B, S_{1}, \ldots, S_{\mathrm{n}}\right)$ entre sistemas relacionales $\left(A, R_{1}, \ldots, R_{\mathrm{m}}\right)$ y $\left(B, S_{1}, \ldots, S_{\mathrm{n}}\right)$ en las que $A$ y $B$ son los dominios de base de los sistemas y $R, S$ las relaciones en ellos. Suppes, Mundy y otros han desarrollado de manera minuciosa la teoría formal de este tipo de aplicaciones preservadoras de estructura y, consiguientemente, aquí podemos considerarla como conocida ( $c f r$. Mundy 1986).

Es importante remarcar que en el caso de las representaciones preservadoras de estructura $(A, R) \stackrel{r}{\longrightarrow}(B, S)$ no existe ninguna semejanza directa entre sus relatores, representado y representante. Éste claramente es el caso en los ejemplos paradigmáticos de la teoría representacional de la medida: entre los objetos empíricos y sus correspondientes números no existe ninguna semejanza. Se puede hablar, sin 
embargo, de una semejanza estructural (parcial) en cuanto que la condición de preservación estructural asegura que una relación asociativa o conmutativa se representa por otra del mismo tipo. Es decir, cuando se representa una relación empírica de orden $\preceq$ o una operación empírica de concatenación $\otimes$ por una relación numérica de orden $\preceq$ o una operación aritmética + de números reales, ello sólo es posible porque se cumplen ciertas condiciones de "semejanza" estructural, a saber, el requisito de que tanto $\preceq$ y $\otimes$ como $\preceq \mathrm{y}+$ son respectivamente transitivos o asociativos y conmutativos. Las propiedades relacionales de las relaciones representadas y representantes deben corresponderse en cierto sentido; puede afirmarse así una semejanza estructural entre el dominio representado y el representante. Esta semejanza estructural entre las relaciones representadas y las representantes es siempre parcial, es decir, pueden fijarse propiedades relacionales para $\otimes$ o para + que carezcan de correspondencia en cualquier otro dominio que no sea el suyo mismo. Las condiciones estructurales que acabamos de definir informalmente singularizan la relación representante entre los dominios $A$ y $B$ como un homomorfismo parcial. Por ello, en lo que sigue denominaremos representaciones homomorfas a las representaciones preservadoras de estructuras.

Este tipo de representación, concebida bajo la forma de semejanza estructural parcial, es el tipo de representación más estudiado en la filosofía de la ciencia. No es, sin embargo, el tipo representacional más utilizado en las ciencias. Avanzamos un paso en el objetivo descrito para el artículo con nuestra presentación de la representación isomórfica: la tesis que queremos sostener es que las representaciones científicas no son generalmente de tipo homomórfico, sino de otro tipo de representación en el que ya no es posible referirse ni a la semejanza objetual ni a la similitud 
estructural. Denominamos a este tipo de representación, representación homóloga o functorial.

(4) La representación como homología

Para comenzar, por lo que acaba de decirse, podemos caracterizar las representaciones homólogas negativamente con relación a otras representaciones: no dependen ni de la semejanza objetual ni de la estructural. De ahí que se alejen de toda concepción de representación concebida como reflejo especular o basada en la noción de semejanza. Constructivamente, la idea de la representación homóloga puede introducirse a partir de algunas consideraciones formuladas por Hertz acerca de la estructura general de las teorías físicas en su obra Prinzipien der Mechanik. Ha de remarcarse que esas consideraciones se usan aquí para fijar el punto de partida de una teoría de las representaciones homólogas. Aunque sugestiva en muchas ideas, la filosofía de la ciencia de Hertz está superada en aspectos esenciales; la asunción anterior no nos compromete con estos últimos.

Hertz postula que el fin esencial de todas nuestras teorías empíricas consiste en realizar predicciones de nuestras experiencias futuras, y se plantea el problema de describir de manera precisa el procedimiento por el cual podemos lograr ese fin:

nos hacemos imágenes (Scheinbilder) internas o símbolos de los objetos externos, y los hacemos de tal manera que las consecuencias intelectualmente necesarias (denknotwendigen) de las representaciones (Bilder) son siempre a su vez representaciones (Bilder) de las consecuencias naturalmente necesarias (naturnotwendigen) de los objetos derivados. Para que esa condición sea completamente satisfecha deben existir ciertas concordancias entre la naturaleza y nuestra mente. La experiencia nos enseña que esa condición es satisfacible y que tales concordancias existen de hecho (Hertz 1894, p. 1). 
Para lo que aquí nos interesa, el aspecto esencial de la descripción de la actividad científica que Hertz hace, radica en describir esa actividad como la producción de una simetría entre las "consecuencias naturalmente necesarias" y las "consecuencias intelectualmente necesarias" de sus "representaciones". La idea de esa simetría puede captarse por un diagrama procesual del siguiente tipo:

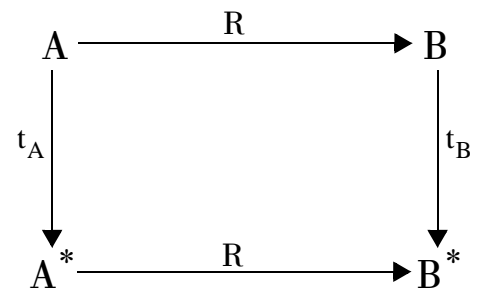

Este diagrama contiene, como indicaba Hertz, la estructura de la argumentación o, mejor aún, de la representación de una teoría empírica. En lo que sigue procederemos a explicitar esta afirmación.

La parte izquierda del diagrama $A \stackrel{t_{\mathrm{A}}}{\longrightarrow} A^{*}$ puede interpretarse como un proceso empírico en el que se pasa del estado $A$ al estado $A^{*}$ a través de $t_{\mathrm{A}}$. Este paso se representa por un proceso teórico consistente en el paso de $B$ a $B^{*}$ por $t_{\mathrm{B}}$, tal que $t_{\mathrm{B}}$ es una relación lógica (o generalizando, matemática) entre $B$ y $B^{*}$ que corresponde a la relación $t_{\mathrm{A}}$ "naturalmente necesaria", en el sentido que el diagrama conmuta. Es decir, en el sentido de que la transición de $A$ por $t_{\mathrm{A}}$ y $R$ conduce al mismo resultado que la transición de $A$ por $R$ y $t_{\mathrm{B}}$, esto es, a $B^{*}$.

Duhem expresa esta relación entre la "necesidad natural" y la "necesidad intelectual" de manera algo más concreta. 
Él indica que un experimento físico, o mejor, la construcción de una experimentación física presenta siempre dos aspectos: "[la construcción consiste en que] el físico compara sin cesar dos instrumentos entre sí, el instrumento real que manipula y el instrumento ideal y simbólico sobre el que razona" (Duhem 1906, p. 236). La "comparación entre ambos instrumentos" se puede explicitar formalmente mediante el diagrama de Hertz. Como Duhem muestra detalladamente en ejemplos concretos, el experimento real y el simbólico se corrigen mutuamente. De hecho, en Duhem puede identificarse ya lo que mucho más tarde Pickering ha descrito como el mecanismo del rodillo ("mangle") de la práctica, es decir, la "dialéctica de resistencia y adecuación” ( $c f r$. Pickering 1995). En el apartado 5 trataremos más detenidamente esta cuestión.

Consideremos ahora la posibilidad de concatenar o iterar de manera natural el diagrama anterior de Hertz del siguiente modo:

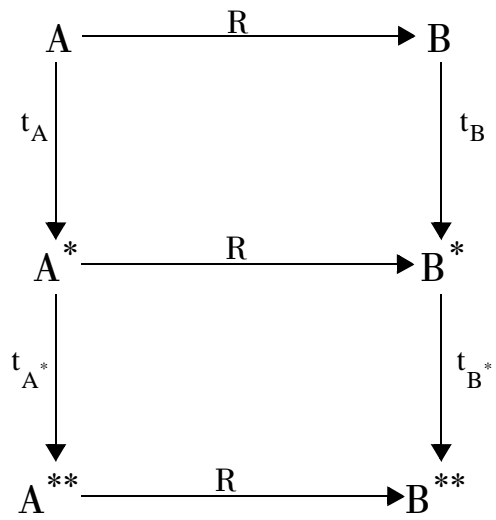


Este diagrama debe cumplir la condición de que "todos los caminos conducen al mismo fin", es decir:

$$
t_{\mathrm{B}^{*}} \cdot t_{\mathrm{B}} \cdot R=t_{\mathrm{B}^{*}} \cdot R \cdot t_{\mathrm{A}}=R \cdot t_{\mathrm{A}^{*}} \cdot t_{\mathrm{A}}
$$

Requerimos además la condición evidente de que un proceso trivial $A \stackrel{i d}{\longrightarrow} A$ consistente en que "nada" sucede en él, tiene como imagen lógica la relación de identidad $B \stackrel{i d}{\longrightarrow} B$. Ésta es una condición mínima por la que se asegura que la "aplicación lógica" de Hertz de la "necesidad natural" no produce ningún artefacto superfluo que no tenga correspondencia en el ámbito de los objetos.

Podemos formular resumidamente ahora las tres condiciones establecidas sobre la representación de Hertz:

(2.3) Condiciones para el diagrama de Hertz

(1) $t \cdot R=R \cdot t$

(2) $R\left(t \cdot t^{*}\right)=R(t) \cdot R\left(t^{*}\right)$

(3) $R(i d)=i d$

Estas tres condiciones explicitan propiedades implícitas del enfoque de Hertz. Lo interesante es observar que (2.3) corresponde exactamente a las condiciones que debe cumplir un functor - entendido en el sentido de la teoría de categorías - definido entre las categorías de A-objetos y B-objetos (Mac Lane 1971, Goldblatt 1979). Podemos concebir, por lo tanto, el diagrama de Hertz como un diagrama functorial que establece una relación de representación entre una A-categoría "empírica" y una B-categoría "simbólica". Un diagrama functorial de este tipo no sólo 
faculta una iteración como la expresada en (2.2), sino que permite además concebirla en la otra dirección, esto es:

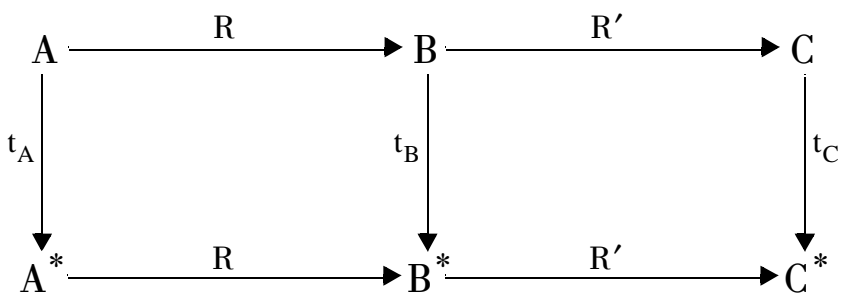

Esta iteración muestra que la distinción entre el dominio "simbólico" y el "empírico" es relativo: es decir, un dominio es simbólico siempre con relación a otro, no en un sentido absoluto. Más adelante veremos que las representaciones iteradas de este tipo desempeñan de hecho un papel fundamental en las ciencias. Una cualidad determinante del enfoque representacional es justamente la posibilidad de presentar esas iteraciones de manera natural.

No nos detendremos aquí en los detalles de la teoría de categorías: nos limitaremos al análisis de las diferencias singularizables entre las representaciones functoriales u homólogas y las representaciones (parcialmente) preservadoras de estructura, las únicas que como se dijo han merecido hasta el momento la atención de los filósofos de la ciencia. En las representaciones homólogas no se requiere, como se exigía en el caso de la aplicación parcialmente preservadora de estructura, que las relaciones $t_{\mathrm{A}}$ y $t_{\mathrm{B}}$ sean del mismo tipo, es decir, que cumplan la condición de la asociatividad, la conmutatividad, la transitividad u otras semejantes. Tampoco se presupone que $A$ y $B$ sean conjuntos y que la representación $r$ sea una aplicación teóricoconjuntista. En este sentido las representaciones homólogas 
semejan a las representaciones vicariales o sustitutivas. La única condición constitutiva que se postula es la de la conmutatividad para el paso de $A$ a $A^{*}$ por $t_{\mathrm{A}}$. Podemos interpretarla por lo tanto como una representación lógica: no son ni los objetos ni las estructuras los que son considerados y representados, sino únicamente las relaciones lógicas, es decir, en nuestro caso, $t_{\mathrm{A}}$ por $t_{\mathrm{B}}$. Este enfoque induce claramente un contextualismo u holismo local.

$\mathrm{Si}$ distinguimos ahora entre la semejanza objetual (Osemejanza), la estructural (E-semejanza) y la lógica (Lsemejanza), el comportamiento de las diferentes formas de representación puede expresarse de manera explícita:

(2.5) Formas de representación.

Se pueden distinguir las siguientes formas de representación respecto de su "comportamiento sobre la semejanza"

\begin{tabular}{l|cccc} 
& Isomorfía & Sustitución & Homomorfía & Homología \\
\hline O-Semejanza & + & - & - & - \\
\hline E-Semejanza & + & $0(-)$ & + & - \\
\hline L-Semejanza & + & $0(-)$ & + & + \\
\hline
\end{tabular}

La concepción intuitiva de la representación como reflejo especular se basa en una isomorfía entre una entidad representada y otra representante. Como muestra (2.5) la representación especular induce una estricta concordancia objetual, estructural y lógica entre los dos relatores de la representación, el ámbito representado y el representante. En los dos niveles intermedios de la tipología de la representación, esto es, en la representación sustitutiva y en la homomorfa, la concordancia de la representación isomórfica - cuya finalidad es la obtención de una mera duplicación de lo representado - se diluye gradualmente. Finalmente, la representación homóloga es la forma de representación más alejada de la representación isomorfa y, como muestra (2.5) se basa únicamente en una semejanza lógica. 
En lo que sigue procuraremos hacer razonable la tesis de que en general las representaciones en la ciencia presentan el formato característico de las representaciones homólogas. Si ello es así, no es preciso requerir como regla general, como lo hace la concepción representacionista dominante, la caracterización de las representaciones científicas como aplicaciones (parcialmente) preservadoras de estructura. En particular, no es necesario sostener una imagen de la teoría científica como una entidad que procura una representación que preserva la estructura de una parte del mundo.

Esta concepción se asocia originariamente al enfoque realista estructural de Helmholtz. Hacia este realismo estructural tienden también actualmente, aunque de manera dispar, las diversas versiones del "semantic view". Van Fraassen por ejemplo postula que las estructuras empíricas pueden concebirse como subestructuras que encajan en estructuras teóricas (van Fraassen 1989); Giere aboga por considerar las estructuras empíricas como "semejantes" a las estructuras teóricas. Estas perspectivas se sostienen claramente en alguna de las versiones de lo que hemos denominado semejanza estructural. Es incuestionable que el énfasis en las representaciones (parcialmente) preservadoras de estructuras caracteriza la bibliografía actual en la filosofía de la ciencia que se ocupa del análisis del concepto de representación (cfr. Suppes 1989, Swoyer 1991, Mundy 1986 o Díez 1998). Este interés ha conducido, por un lado, al desarrollo de una impresionante teoría, cuyos signos más remarcables están contenidos en la teoría representacionista de The Foundations of Measurement (Krantz et al. 1971-1990), pero por otro lado ha inspirado una postura reductiva, por la cual todas las representaciones de la ciencia están conformadas según el modelo de las representaciones parcialmente preservadoras de estructuras.

Nuestro enfoque procura un concepto de representación más general, el concepto de representación functo- 
rial u homóloga (en el sentido de la teoría matemática de categorías). Trataremos de mostrar que esta concepción homóloga de la representación es esencialmente más flexible que la representación inspirada en el representacionismo estructural de raíz helmholtziana.

\section{Aspectos combinatorios de las representaciones}

Sea cual fuere el tipo de representación sometido a escrutinio, es decir, trátese de representaciones sustitutorias, especulares o functoriales, una característica común a todas ellas es que no coexisten de manera independiente, sino que forman una compleja red representacional. En esa red pueden identificarse distintos tipos de combinaciones entre las representaciones.

Por ejemplo, puede ocurrir que una entidad $A$ se represente por distintas entidades $B, C, D$, etc., de suerte que tengamos distintas relaciones representacionales a partir de la misma fuente: $A \stackrel{r}{\longrightarrow} B, A \stackrel{s}{\longrightarrow} C, A \stackrel{t}{\longrightarrow} D$. A la inversa, puede ser también que una misma entidad $E$ sea la entidad representante para diversos objetos $A, B, C$, etc., de manera que nos encontremos con relaciones representacionales tales como $A \longrightarrow E, B \longrightarrow E, C \longrightarrow E$, etc. Es posible además encontrar iteraciones representacionales que para las representaciones $A \stackrel{r}{\longrightarrow} B$ y $B \stackrel{s}{\longrightarrow} C$ definan por ejemplo una representación indirecta o compuesta $A \stackrel{s \cdot r}{\longrightarrow} C$. Puede suceder finalmente que en algunos contextos determinados una entidad $A$ se represente a sí misma: $A \stackrel{i d}{\longrightarrow} A$. Éste no es un caso degenerado; expresa por ejemplo la posibilidad existente en muchos sistemas jurídicos de que un acusado se defienda a sí mismo ante un tribunal.

Esta sencilla presentación de diversas posibilidades combinatorias entre las representaciones es suficiente para requerir de una teoría formal de las representaciones, una 
componente combinatoria que describa la combinación o reiteración posible de las distintas representaciones. En lo que sigue procuraremos justificar esta afirmación.

Es importante remarcar, para comenzar, que las combinaciones representacionales pueden realizarse tanto sobre representaciones preservadoras de estructura como sobre las homólogas. Por lo tanto, no es preciso distinguir en principio entre ambos tipos de representaciones para introducir unas primeras reflexiones combinatorias. Así, en ambos tipos de representaciones se cumple que las representaciones $f: A \longrightarrow B, g: B \longrightarrow C$ pueden combinarse como $A \stackrel{g \cdot f}{\longrightarrow} C$. Puede admitirse además que esa combinación o concatenación es asociativa, esto es, que se cumple la ley de la asociatividad en las representaciones que $f, g$ y $h$ "realizan entre si":

$$
f \cdot(g \cdot h)=(f \cdot g) \cdot h .
$$

Esta combinación o iteración de representaciones es de una importancia central en la práctica científica general, aunque quizás por su generalidad resulte frecuentemente trivializada. Así, por ejemplo, la medida numérica de un dominio empírico, que se expresó anteriormente por $r$ : $D \longrightarrow R$, es en realidad, observada bajo un escrutinio más fino, una cadena más o menos larga de representaciones:

$$
D \longrightarrow E \longrightarrow F \longrightarrow \ldots \ldots \longrightarrow R
$$

Las representaciones numéricas o, en general, las representaciones matemáticas de los objetos de $D$ sólo se pueden "realizar" de manera directa en unos pocos casos; generalmente son constructos que se producen a lo largo de un proceso más o menos complejo de constitución. Esa complejidad típica de las representaciones científicas ha sido resaltada por autores como Galison (1997), Pickering (1995), Laymon (1987) y otros. Los distintos tipos de elaboración y procesamiento de representaciones - digitalización, analo- 
gización, etc. - desempeñan una función importante en la conformación de esas complejas representaciones. El largo recorrido identificable entre los datos y la teoría muestra entonces que la dicotomía estándar establecida entre un dominio teórico y otro empírico es, en el mejor de los casos, una imagen muy idealizada de la ciencia real; una imagen necesitada, en cualquier caso, de concretización.

Un paso en esa dirección consiste en reconocer la larga distancia existente entre los datos y los constructos teóricos y tratar de describirla de manera más detallada. Así lo hacía, por ejemplo, Laymon (1982) al describir el largo "camino contrafáctico de los datos a la teoría" de la teoría general de la relatividad. Hemos seguido esa estrategia en (Ibarra/Mormann 1994, 1998) tratando de explicitar ese camino como una cadena de representaciones. En (Ibarra/Mormann 1998), introdujimos la distinción entre datos (D), fenómenos (P) y constructos teóricos (T), de forma que el dominio jerárquicamente estructurado de fenómenos (P) funciona como un ámbito de mediación entre los datos "brutos" y los constructos teóricos:

$$
\mathrm{D} \Longrightarrow(\mathrm{P}) \Longrightarrow \mathrm{T}
$$

Otra estrategia posible en la explicitación del reductivo y opaco modelo del doble nivel $\mathrm{D} \Longrightarrow \mathrm{T}$, consiste en relativizar, o mejor, en tomar conciencia del status relativo de los dos "valores-límite" o "puntos finales" -D,T- de ese camino. Tenemos así una serie (potencialmente) infinita de representaciones:

$\ldots E_{-\mathrm{n}} \longrightarrow E_{-(\mathrm{n}-1)} \longrightarrow \ldots \longrightarrow E_{-1} \longrightarrow E_{0} \longrightarrow E_{1} \longrightarrow E_{2} \longrightarrow \ldots$

Éste es el modelo que presenta Latour para tratar de superar de algún modo el "abismo" existente entre el "mundo" y el "lenguaje" ( $c f r$. Latour 1999, pp. 69ss). Su "deam- 
bulatoria" conceptualización de la referencia (deambulatory conception of reference) queda fijada por una cadena de representaciones, cuyos elementos, como en (3.4), pueden interpretarse como "pequeñas" representaciones. Latour analiza conspicuos estudios de casos que documentan detalladamente el largo camino del "mundo" al "conocimiento". Este planteamiento no es completamente novedoso. Se encuentra ya en Cassirer (Cassirer 1910), cuando se enfrenta a la estricta contraposición entre "pensar" y "ser", caracterizándola como un artilugio aporético de una metafísica trasnochada ( $c f r$ r también, James 1907).

Ahora bien, aun cuando en sentido estricto la constitución del conocimiento científico se describa como una serie de representaciones, en determinadas circunstancias puede resultar de interés anclar los extremos de esa serie de manera "sintetizadora" o al modo de una caja negra. Se obtiene así una serie del tipo (3.3) como la estudiada en (Ibarra/Mormann 1998). Otra idealización posible consiste en reducir la cadena $D \longrightarrow \ldots \longrightarrow C$ a sus elementos finales. Se alcanza entonces el conocido modelo de los dos niveles $D \longrightarrow C$ imperante hasta hoy en la filosofía de la ciencia (Ibarra/Mormann 1998). Así pues, una concepción representacional es un candidato razonable para el carácter serial del conocimiento científico, en cuanto que, tal como lo entendemos, en el concepto de representación se considera primariamente la posibilidad de combinación de las representaciones.

La combinabilidad de las representaciones no se limita sin embargo al tipo de combinaciones lineales que Cassirer y Latour examinan. La teoría matemática de categorías es de hecho una teoría de la combinación de representaciones, en la cual éstas se caracterizan como morfismos o functores. Más concretamente, la teoría de categorías es una teoría de combinaciones posibles. Ella ofrece un abundante instrumental conceptual y metodológico para ser usado en la 
investigación de las combinaciones representacionales. No entraremos aquí en el estudio de ese inventario. Consideraremos dos o tres ejemplos sencillos, en los que se procurará mostrar la significación epistemológica de la teoría de categorías concebida como una teoría combinatoria general de las representaciones. Esos ejemplos comprenden fundamentalmente combinaciones no lineales de representaciones.

Normalmente una representación $A \longrightarrow B$ es insuficiente para determinar científicamente un dominio $A$. Puede ocurrir que $A \longrightarrow B$ sea tan burda que en la $B$ representación de $A$ se pierdan aspectos esenciales de $A$. De ahí que se intente construir, junto con $A \longrightarrow B$, otras representaciones $A \longrightarrow B^{*}, A \longrightarrow B^{* *}$, etc. La totalidad de esas representaciones tiene que ser compatible de alguna manera que hay que precisar. Para empezar, esta compatibilidad no es por sí misma una condición suficiente: las diversas representaciones $A \longrightarrow B, A \longrightarrow B^{*}$ no deben ser meras repeticiones. La totalidad de las diversas representaciones debe estar constituida de tal manera que ofrezcan en conjunto una representación más rica de $A$ que la que ofrecen individualmente.

Por mor de la sencillez consideraremos sólo dos representaciones distintas, $A \stackrel{r}{\longrightarrow} B$ y $A \stackrel{r^{*}}{\longrightarrow} B^{*}$. Lo que pretendemos es obtener una representación $A \stackrel{r_{\mathrm{p}}}{\longrightarrow} P$ que sintetice toda la información (lógica) sobre $A$, que hemos conseguido por $r$ y $r^{*}$, de manera que "nada se pierda". Si tal representación existe - puede ocurrir que $r$ y $r^{*}$ sean incompatibles-, esa representación es justamente el producto de $B$ y $B^{*}$. Podemos definirlo representacionalmente: ese producto es una "representación doble" $B \stackrel{P}{\longleftrightarrow} B^{*}$ tal que para todo $A$ existe una representación única $A \longrightarrow P$ que verifica la conmutatividad del diagrama: 


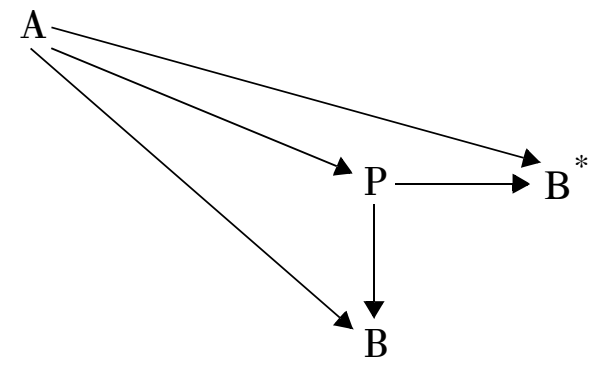

Esta presentación abstracta del producto puede resultar algo extraña: si $B$ y $B^{*}$ son conjuntos (estructurados), $P$ es justamente el producto cartesiano $B \times B^{*}$ y es trivial que si $B$ y $B^{*}$ existen, existe también el producto $B \times B^{*}$. La cuestión crucial aquí radica, sin embargo, en que una teoría general de la representación no puede postular que todas las representaciones sean de ese tipo. Consideremos que $B$ y $B^{*}$ sean máquinas, es decir, sistemas experimentales materiales, empíricos, que miden diversos aspectos de $A$. En este caso, como muestra la teoría cuántica, no existe un producto de las representaciones del tipo que acabamos de presentar.

Consideremos como segundo ejemplo una combinación de representaciones que de alguna manera sea complementaria del producto. Sean dos representaciones competidoras $A \stackrel{f}{\longrightarrow} B$ y $A \stackrel{g}{\longrightarrow} B$ y admitamos además que negligimos los aspectos que las diferencian. Esto es lo que sucede por ejemplo cuando consideramos $f$ y $g$ como realizaciones del mismo experimento, relegando las diferencias que pudieran existir. En este caso buscamos una representación que desatienda las diferencias existentes entre $f$ y $g$, pero que no pierda ninguna información más. Es decir, en el lenguaje diagramático de una teoría combinatoria general de 
las representaciones nos preguntamos si existe una representación $q: B \longrightarrow E$ que verifique la conmutatividad del diagrama:

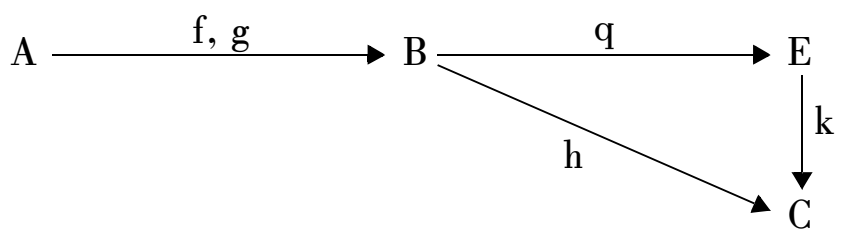

Si $h: B \longrightarrow C$ es la representación que desatiende la diferencia entre $f$ y $g$, esto es $h \cdot g=h \cdot f$, existe un $k$ : $E \longrightarrow C$ tal que $h$ factoriza sobre $q$ en el modo indicado. Esto significa que los agregados $h \cdot g$ y $h \cdot f$ de $g$ y $f$ pierden al menos tanta información como los agregados $q \cdot g$ y $q \cdot f$. Por lo tanto, tampoco aquí se requiere que exista un filtro óptimo. Si existiera se definiría como coigualador de $g$ y $f$. Es sencillo construir un filtro así para la aplicación teóricoconjuntista $f$ y $g$; en las representaciones materiales, sin embargo, es evidente que no se necesita la existencia de tal filtro.

Estos ejemplos son suficientes para hacer posible nuestra tesis de que no son únicamente las combinaciones lineales las que desempeñan una función esencial en una teoría representacional del conocimiento científico; también lo desempeñan, y en mayor grado aún, las combinaciones nolineales. En los dos apartados siguientes vamos a probar en dos ejemplos concretos la aplicabilidad del aparato de una teoría combinatoria de la representación, que hasta ahora sólo se ha esbozado en sus lineamientos más generales. 


\section{El grupo fundamental de Poincaré}

Luego de haber examinado con cierto detenimiento el aparato técnico formal de las representaciones homólogas, es tiempo de considerar al menos algunos ejemplos que muestren la aplicación del método de la representación homóloga en la práctica científica.

Elegimos para ello dos ámbitos suficientemente alejados entre sí: uno del dominio de la topología, el otro del de la actual física de partículas. La elección no es circunstancial: nos permite postular implícitamente que el concepto delineado de representación homóloga es aplicable a todos los dominios científicos. En otras palabras, sostenemos la tesis de que existen analogías esenciales entre la matemática y la ciencia empírica, sobre la base de que ambos dominios del conocimiento pueden caracterizarse como ejemplos genuinos de actividad representacional homóloga. Esta afirmación debe cualificarse. No afirma que esa actividad representacional se realice en general bajo la forma de producción de representaciones matemáticas; asevera más bien que las representaciones en la matemática muestran las características típicas de las representaciones homólogas que encontramos en todos los dominios de las ciencias.

Comenzamos con el ejemplo de la topología. El grupo fundamental de Poincaré se define intuitivamente con relativa sencillez, lo que lo hace especialmente apropiado para la introducción a la teoría de las representaciones homólogas. El grupo fundamental de Poincaré se define tanto para objetos geométricos (como las variedades), como para espacios topológicos; por ejemplo, circunferencias, toros, o variedades espacio-temporales. Pues bien, sea $M$ una variedad, elegimos un punto base en $M, m \in M$, y tomamos todos los bucles que comienzan y terminan en $m$. Esto es, matemáticamente hablando, un bucle así es una 
aplicación continua $s: I \longrightarrow M$ del intervalo $I=[0,1]$, con $s(0)=s(1)=m$ :

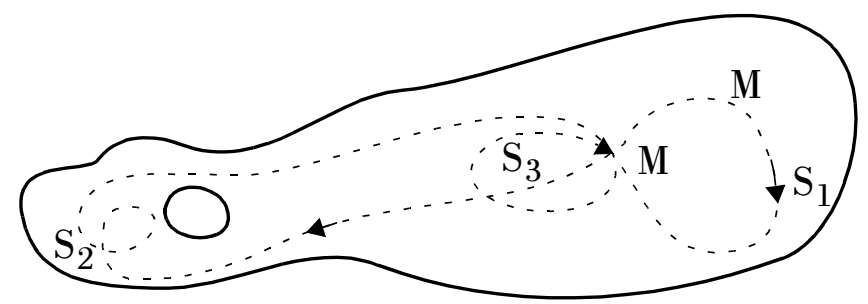

Podemos "sumar" bucles de este tipo, de manera que la adición $s_{1}+s_{2}$ de $s_{1}$ y $s_{2}$ se defina como el bucle que recorre primero $s_{1}$ y después $s_{2}$. Prescindamos de detalles técnicos y supongamos que $M$ es suficientemente conexa. Resulta entonces que en la clase de los bucles de $M$ que comienzan y terminan en $m$ se puede definir una adición que hace a esa clase un grupo en sentido algébrico. Ese grupo es independiente del punto base elegido contingentemente, y en consecuencia es una invariante de $M$. Denominamos a esa invariante grupo fundamental (de Poincaré) —o primer grupo de homotopía- de $M$ y lo designamos por $\pi_{1}(M)$. Es fácil ver que una aplicación (continua) $f: M \longrightarrow N$ de la variedad $M$ a la variedad $N$ induce un homomorfismo de grupo $\pi_{1}(f): \pi_{1}(M) \longrightarrow \pi_{1}(N)$ que hace corresponder a los bucles de $M$ bucles en $N$. Es decir, $\pi_{1}$ es un functor - en el sentido de la teoría de categorías - que asigna categorías de grupos a las categorías de variedades ( $c f r$. Mac Lane 1971). A la representación que representa variedades geométricas por sus grupos fundamentales la denominamos representación functorial. 
Veamos entonces esquemáticamente en qué sentido puede interpretarse $\pi_{1}(M)$ como una representación homóloga para variedades y espacios topológicos. El diagrama de Hertz

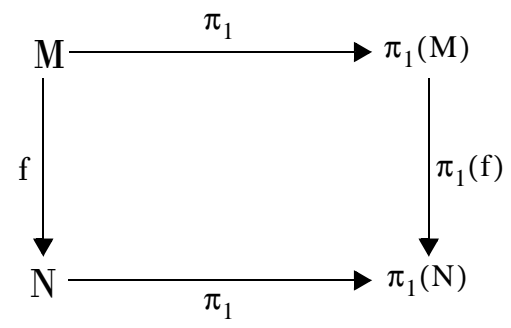

prueba que la correspondencia del grupo fundamental $\pi_{1}(M)$ a una variedad $M$ es una representación homóloga.

Como sugiere la denominación de "primer grupo de homotopía" para $\pi_{1}(M)$, existen otros "grupos de homotopía más elevados", que los designamos como $\pi_{n}(M), n>0$. No entraremos aquí en la definición de esos grupos de homotopía; basta con indicar que son también invariantes de $M$, que en general se estudian conjuntamente con $\pi_{1}$, y que naturalmente se interpretan también como representaciones homólogas.

El problema general de la determinación de los grupos de homotopía como representantes homólogos de variedades es que para una variedad dada $M$ no existe en principio ninguna posibilidad efectiva de calcular realmente sus grupos de homotopía. Incluso para variedades muy "simples" como las circunferencias no se conocen por el momento todos sus grupos de homotopía. Este desconocimiento es de calado más profundo que el desconocimiento detectable 
en otros ámbitos, como por ejemplo, la ignorancia relativa a la secuencia decimal de los números irracionales. Aunque desconozcamos la cifra correspondiente a la $10^{10}$-sima posición de $2^{1 / 2}=1^{\prime} 414 \ldots$, existe un algoritmo que permite calcularla en un tiempo prudencial. Por el momento esto no es posible para el cálculo de los grupos de homotopía.

En el cálculo de estos grupos $\pi_{n}$ no sólo se someten a detallado escrutinio las propiedades específicas de la variedad en cuestión; en el examen matemático se introducen también grupos de homotopía ya conocidos de otras variedades. Es decir, si se desean calcular los grupos de homotopía de una variedad $M$, debe encontrarse para ella una red apropiada de otras variedades cuyos grupos de homotopía son ya conocidos —al menos parcialmente. Este conocimiento junto con el de otros medios generales de cálculo es utilizado para determinar los grupos de homotopía de $M$. Tal práctica semeja a la de un experimento calculatorio, cuya construcción es con frecuencia extremadamente compleja: el objetivo es producir una configuración en la que se puedan calcular los grupos de homotopía de $M$ paso a paso. Este procedimiento guarda semejanza con el seguido en la resolución de un crucigrama, en el que se trata también de extender paso a paso el conocimiento mediante sutiles aplicaciones de conocimiento parcial (cfr. Haack 1993).

Sólo se conocen a priori los grupos de homotopía de unas pocas variedades; entre ellas los de los espacios ndimensionales $R^{\mathrm{n}}, \mathrm{n} \succeq$. Para éstos se trata de probar que $\pi_{\mathrm{i}}\left(R^{\mathrm{n}}\right)=0$ para todo $i$. Ya en el caso de las circunferencias $S^{\mathrm{n}-1} \subseteq R^{\mathrm{n}}$ no se han calculado todavía todos sus grupos de homotopía. La única excepción es $\mathrm{S}^{1}$ : para su cálculo se cuenta con la circunstancia de que la aplicación exponencial $x \longrightarrow e^{\text {ix }}$ define una aplicación continua e: 
$R^{1} \longrightarrow S^{1}$ que permite finalmente calcular $\pi_{1}\left(S^{1}\right)=\mathbb{Z}$, siendo triviales todos los grupos de homotopía más altos de $S^{1}$.

Por supuesto el cálculo de abstrusas invariantes topológicas de objetos geométricos no es ningún fin en sí mismo. Es legítimo preguntarse para qué sirven todas esas preocupaciones calculatorias. La respuesta está en línea con la función general atribuida a las representaciones: sirven porque facultan una forma de razonamiento subrogatorio. Es decir, porque la imagen homóloga de los grupos de homotopía permite derivar conclusiones respecto de las variedades y sus relaciones geométricas.

Un ejemplo del rendimiento de la representación homóloga del grupo fundamental es el teorema del punto fijo de Brouwer. El problema del punto fijo de una variedad $M$ consiste en determinar si existe una aplicación $f: M \longrightarrow M$ que no tenga ningún punto fijo, esto es, para la que se cumple $f(x) \neq x$ para todo $x$ de $M$. Tal aplicación sin punto fijo sería tan distinta como posible de la aplicación de identidad $i d: M \longrightarrow M$, que establece una correspondencia de cada punto consigo mismo $(i d(x)=x)$. El problema del punto fijo de Brouwer es decidir si existe una aplicación del círculo $f: D^{2} \longrightarrow D^{2}$ tal que haga corresponder al borde $S^{1}$ de $D^{2}$ de manera idéntica a sí mismo, pero que no tenga ningún punto fijo dentro de $D^{2}$. Si existe tal aplicación ello quiere decir, como fácilmente puede probarse ( $c f r$. Lawvere/Schanuel 1995), que existe una aplicación $r: D^{2} \longrightarrow S^{1}$, de tal modo que la concatenación $S^{1} \stackrel{i}{\longrightarrow} D^{2} \stackrel{r}{\longrightarrow} S^{1}$ es precisamente la identidad. Tenemos así el diagrama de Hertz de doble nivel: 
$(4.1)$

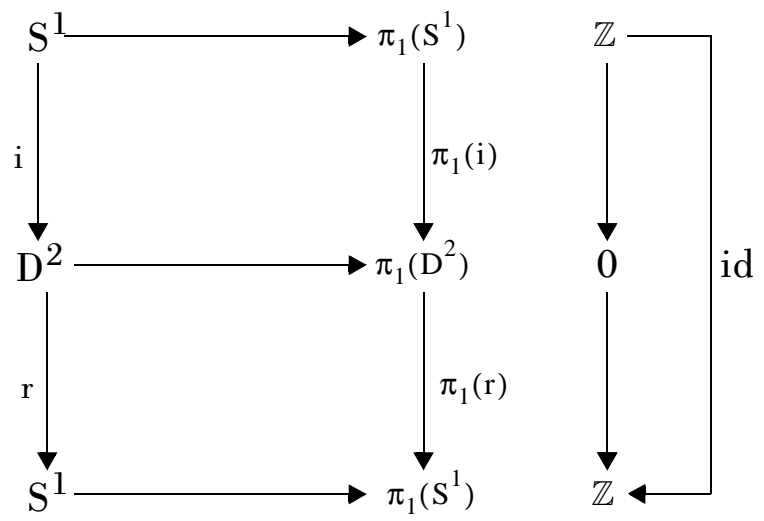

Es claro, sin embargo, que esto no es posible, porque la aplicación idéntica de los números enteros no está factorizada sobre 0. Esta prueba del teorema del punto fijo de Brouwer, que hace un uso esencial de la representación homóloga de las variedades por sus grupos fundamentales, es un sencillo ejemplo de aplicación de la representación homóloga. En cierto sentido este procedimiento es arquetípico y constitutivo del estilo de la matemática del siglo XX (cfr. Lawvere/Schanuel 1995).

5. Representaciones homológicas en la ciencia empírica

Consideraremos ahora algunos ejemplos de representaciones homólogas en la ciencia empírica y más concretamente, como se dijo, en la física de partículas actual. Análogamente al caso de las teorías matemáticas, nuestro punto de partida es también aquí el diagrama de Hertz: 


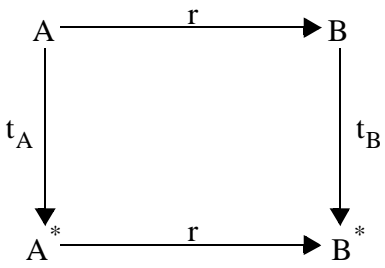

Ahora bien, mientras que para la matemática la concepción filosófica habitual tiende a ignorar las diferencias entre el A-dominio y el B-dominio, esa misma concepción propende en el caso de las ciencias empíricas a enfatizar la diferencia entre ambos dominios. Una presentación clásica de esa diferencia se encuentra en la obra de Duhem, La teoría física, su objeto, su estructura (Duhem 1906, part. II, cap. 4). En ella su autor insiste enfáticamente en que existe una diferencia esencial entre un "hecho práctico" y un "hecho teórico, o sea, simbólico y formal", que hace imposible una traducción directa entre ambos. Esto es debido sobre todo a que "a un mismo hecho teórico puede corresponder una infinidad de hechos prácticos distintos", y a la inversa, a que "a un mismo hecho práctico puede corresponder una infinidad de hechos teóricos lógicamente incompatibles" (p. 229).

Los nuevos enfoques de la filosofía de la ciencia orientados hacia el estudio de la práctica experimental de las ciencias empíricas —Galison (1997), Gooding (1990), Latour (1999) o Pickering (1995) - remarcan la dificultad de la construcción de un diagrama de Hertz, esto es, la dificultad de constituir un equilibrio entre práctica, instrumentación, interpretación y teoría. Esos enfoques insisten en que en todo caso esa construcción es el fin explícito de toda actividad científica. Si reformulamos en nuestros 
términos la concepción del "rodillo" de Pickering como una dialéctica de resistencia y adecuación, podemos caracterizar la investigación científica como un proceso que parte de un diagrama no-conmutativo en el que por lo tanto no coinciden los caminos $t_{\mathrm{B}} \cdot r$ y $r \cdot t_{\mathrm{A}}$, y cuyo objetivo es tratar de encontrar un diagrama conmutativo. De manera más precisa, la dialéctica del rodillo consiste, en primer lugar, en reemplazar el diagrama no-conmutativo por otro en el que tanto el A-lado empírico como el B-lado simbólico se modifiquen de manera apropiada para lograr verificar la conmutatividad de un diagrama. El desarrollo paso a paso de este cambio lo ha analizado detalladamente Pickering en su estudio de caso "Facts. The Hunting of the Quark" (Pickering 1995, cap. 3).

Este estudio describe los experimentos llevados a cabo por un grupo de físicos italianos a lo largo de dos décadas para demostrar la existencia de quarks libres. Demostrarla habría supuesto una confirmación contundente de la teoría del camino de las ocho secciones ("eightfold way") desarrollada por Gell-Mann y otros. A tal efecto ese grupo de físicos elaboró una serie de experimentos que, en líneas generales, podrían interpretarse como refinamientos del experimento de Millikan para determinar la carga e del electrón. La propiedad esencial de los quarks predicha por la teoría de Gell-Mann era que su carga debía ser $1 / 3$ o $2 / 3$ de la carga del electrón e. Una confirmación de esa hipótesis requirió construir un experimento análogo al de la gota de aceite de Millikan, para poder identificar así partículas con cargas 1/3 e o $2 / 3$ e. Aunque la precisión de la medición en los experimentos fue finalmente diez millones mayor que la del experimento de Millikan, no se logró medir partículas con carga partida. Con ello se excluían las versiones de la teoría de quarks que admitían la presencia libre de éstos (en circunstancias normales). 
Según Pickering, esta interpretación de tales experimentos revela una relación bastante directa aún entre los resultados empíricos y las hipótesis teóricas. Normalmente, sin embargo, esa relación es mucho más indirecta y se presenta mediante un diagrama iterado del tipo (2.4). Como concluye Pickering, para mediar entre los dos niveles, empírico y teórico, se requieren con frecuencia "cadenas representacionales" (Pickering 1995, 96ss, cfr. también Latour 1999, pp. 69ss).

La presencia de esas cadenas hace naturalmente más compleja la dialéctica del rodillo: las adecuaciones y resistencias se presentan en distintos lugares del diagrama representacional y deben estar respectivamente coordinadas entre sí. En (Gooding 1992) se procura desarrollar un lenguaje diagramático para describir estas complejas cadenas de representación, tomando como base el ejemplo mencionado de la búsqueda experimental de quarks y los experimentos clásicos de Faraday sobre los distintos problemas del electromagnetismo. La concepción de Gooding tiene ciertas semejanzas con la teoría combinatoria de las representaciones que hemos esbozado en el apartado 3. En particular, como ha quedado advertido en el diagrama de Hertz, observa también una distinción relativa entre los dos niveles, simbólico y empírico ("material"):

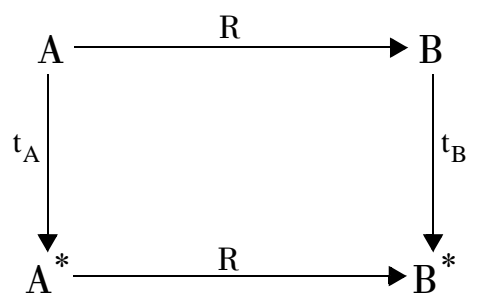


En un lado (A) tenemos, según los términos de Duhem ( $c f r$. Duhem 1906, part. II, cap. 4, §3,4), un instrumento real, esto es, la construcción del experimento real, mientras que en el otro lado (B) incluimos el instrumento simbólico y el resultado experimental simbólico. Ambos lados se conciben inmersos en un proceso de interactividad recíproca. Sólo para la primera fase del experimento. Gooding distingue no menos de seis aparatos distintos $A_{1}, \ldots, A_{6}$. También él, como se sostiene en este trabajo, desestima las relaciones lineales entre los ámbitos conceptual y material. Pero a pesar de estas y otras afinidades, se pueden singularizar importantes diferencias entre el enfoque de Gooding y nuestra teoría combinatoria de las representaciones. Por un lado, el enfoque de Gooding no toma en consideración el concepto central del diagrama conmutativo de Hertz, teorizado de manera precisa en la teoría matemática de categorías. Por otro lado, sus diagramas pueden representar aspectos específicos de la actividad científica representacional, que no son capturables por nuestros diagramas "categoriales". Por ello, la diagramática que se presenta en este trabajo ha de ser entendida claramente como un primer paso en la dirección de la representación metacientífica, esto es, filosófica, de las representaciones científicas.

Otra fuente extraordinariamente relevante para una semántica y pragmática representacionales de las teorías empíricas es la monumental obra de Galison Image and Logic (Galison 1997). Tomando como ejemplo la "cultura material de la microfísica", Galison elabora la significación universal de las representaciones materiales en el desarrollo de la teoría de partículas (subatómicas). En esa obra se distingue entre representaciones "homomorfas" y "homólogas" (Galison 1997, p. 191). Las representaciones homomorfas, tales como las que se producen mediante configuraciones instrumentales como las cámaras de Wilson y de burbujas, ofrecen representaciones "gráficas" de las 
partículas elementales y sus interacciones. Las representaciones homólogas o lógicas son, en cambio, aquellas cuyo objetivo consiste en "[determinar] las relaciones lógicas entre determinadas circunstancias: la partícula no penetró la lámina de hierro 3 pero pasó a través de las láminas de hierro 4, 5 y 6 . Como este modo de registro preserva la relación lógica entre los acontecimientos, lo llamaré representación 'homóloga"” (Galison 1997, p. 19).

Así pues, a diferencia de la representación homomorfa la representación homóloga o lógica no tiene por objetivo ofrecer una imagen "realista" de la realidad subatómica, como la que se supone por ejemplo a través de las imágenes sumamente sugerentes de una cámara de burbujas. Lo que el enfoque (homo)lógico pretende más bien es producir una "imagen lógica del mundo", que pueda caracterizarse mediante el control y la manipulabilidad más completos posibles del aparato experimental. No discutiremos aquí si la distinción que Galison introduce entre "imagen" y "lógica" es adecuada. De ningún modo podemos tratar de adentrarnos en el reino de los hechos fascinantes que Galison ha coleccionado en Image and Logic. Ha de constatarse en todo caso que este libro reúne una impresionante cantidad de material de apoyo para un enfoque representacional.

En resumen, los enfoques que acaban de presentarse coinciden en reconocer la necesidad de un concepto más complejo y plural de representación para describir la compleja y plural práctica de las representaciones científicas.

\section{La ciencia como representación}

Recapitulando el examen de los apartados anteriores podemos afirmar que la ciencia, es decir, la práctica de las ciencias, puede concebirse como una práctica representacional. Esto equivale a caracterizar la práctica de las/os científicas/os como un complejo de actividades de construc- 
ción, combinación, interpretación, procesamiento y "manipulación" de representaciones. Éstas pueden ser de tipos muy distintos: nos encontramos, por un lado, con las representaciones formales de la lógica y la matemática, por otro, con representaciones materiales de molde diverso que desempeñan una función indispensable en las ciencias empíricas; nos topamos además con representaciones língüísticas o proposicionales, etc. Todas estas representaciones pueden a su vez conectarse de formas muy variadas, de tal manera que conforman una enorme red representacional, cuya estructura compete estudiar a la filosofía de la ciencia.

La cuestión central de nuestra concepción diagramática consiste en hacer admisible la idea de que todas esas distintas transformaciones y representaciones pueden capturarse en el diagrama de Hertz (o sus derivados). El argumento esencial para ello es observar que tanto las representaciones conceptuales como las materiales son iterables y que cumplen la ley de la asociatividad $f \cdot(g \cdot h)=(f \cdot g) \cdot h$. Esta ley puede parecer a primera vista demasiado simple y superficial. Las aplicaciones de la teoría de categorías en el dominio de la matemática muestran sin embargo que tiene consecuencias sorprendentemente profundas.

El reconocimiento de la enorme red conexa de representaciones que constituye el dominio de las ciencias no significa que suscribamos un holismo desenfrenado y sin reserva del tipo "todo está conectado con todo en algún sentido u otro". Puede que esta idea sea correcta, pero si lo es, no es muy interesante. Al estudio filosófico corresponde la realización de distinciones. En concreto, el diseño de una teoría combinatoria de las representaciones de naturaleza holista atemperada impone como una primera tarea la identificación de distintas áreas en ese espacio representacional científico de naturaleza reticular. Esa identificación, por otro lado, plantea de manera natural algunas importantes 
reconceptualizaciones en la filosofía tradicional. Mencionaremos unas pocas.

Tradicionalmente se distingue entre el ámbito de los datos y el de los constructos simbólicos, o por decirlo de otra manera, entre "naturaleza" y "teoría". Pero como trató de mostrarse anteriormente, esa distinción es demasiado simple. En línea con los mencionados enfoques de Galison, Gooding, Latour, Pickering y otros, la teoría combinatoria de las representaciones científicas lleva a plantearnos nuevas e interesantes cuestiones ontológicas acerca de la "realidad" de las entidades que forman las cadenas representacionales y, más en general, la red representacional. No puede responderse mediante un escueto "sí" o "no" a la cuestión de si esas entidades representacionales identificables en la red existen o no. Debemos resistirnos, por un lado, a la tentación de adoptar una postura instrumentalista primaria, según la cual los datos serían las únicas entidades "realmente" existentes y los constructos simbólicos símbolos "realmente" no-existentes inventados para procurar predicciones. Por otro lado, una entidad representacional como el electrón no tiene ciertamente el mismo status óntico que, por ejemplo, el renombrado árbol del filósofo. Nuestro enfoque tiende a definir el universo como la totalidad de las entidades representacionales. Esta perspectiva induce una ontología abierta que contrasta claramente, por ejemplo, con la austera ontología positivista que sólo aprueba la existencia de los datos. Esta toma de postura positivista puede ser sugerente por razones de índole filosófica o estética, pero es insostenible si se la somete al juicio de la realidad de la física. Las teorías representacionales están sujetas a una ontología compleja que no puede reducirse a la filosóficamente atractiva pero irrealista simplicidad ontológica tradicional. En el enfoque representacional combinatorio la vieja cuestión del realismo adquiere una nueva modulación: la realidad es nuestra realidad. Ello no equiva- 
le a apoyar un relativismo o idealismo ingenuo que aliente la creencia de que cada uno tiene libertad para inventarse su propio mundo. La consecuencia que se deriva de nuestra toma de postura induce más bien un realismo contingente (cfr. Pickering 1995, Hacking 1999).

Una segunda cuestión a remarcar es relativa a las ramificaciones epistemológicas del enfoque sobre la representación derivadas de su lineamiento peirceano. Las representaciones no están simplemente ahí; más bien, alguien las construye para ciertos fines. Consideraremos brevemente dos objetivos complementarios de las representaciones: (a) la reducción de la complejidad, y (b) la inducción de la complejidad. El enfoque reduccionista de la representación sostiene que la función prevaleciente de la representación consiste en reducir la complejidad superflua. Los ejemplos que pueden aportarse en esa dirección son numerosos: por ejemplo, si deseamos buscar un libro en la biblioteca, no trataremos de encontrarlo directamente en las estanterías, buscándolo al azar, sino que consultaremos el fichero de la bilioteca en el que los libros están representados por fichas o artilugios más avanzados. El fichero puede considerarse claramente como una representación de la biblioteca. Existe una relación fiable entre los libros reales, esto es, el contenido de la biblioteca, y las correspondientes fichas del fichero. Esta representación está claramente motivada por el propósito de reducir la complejidad innecesaria: para encontrar el libro no necesitamos saber su contenido sino únicamente su autor, el título y alguna otra información relevante para encontrarlo. Las fichas son signos que representan a los libros; no necesitan ninguna atribución de semejanza con ellos (Schlick 1918, p. 79). El fichero es una representación que representa los libros atendiendo únicamente a los aspectos relevantes para encontrarlos en los estantes. 
Según esta conceptualización de la representación las entidades representantes se usan como sustitutos o subrogadores de las entidades representadas. Por alguna razón no podemos manipular directamente los objetos originales, lo podemos hacer con dificultad, o creemos conveniente no operar con ellas; en su lugar manipulamos sustitutos apropiados. Es en este sentido como el razonar representacionalmente puede interpretarse como un tipo característico de razonamiento subrogatorio (Swoyer 1991). Ejemplos particulamente relevantes de este tipo de razonamiento son las representaciones y simulaciones numéricas o, en general, matemáticas. Son claras las ventajas de calcular o simular los efectos de una colisión con la ayuda de algún intrumento de representación, en lugar de proceder a preparar una prueba en vivo de la colisión.

Pero aunque el enfoque reduccionista capta algunos aspectos importantes de la representación, sólo describe la mitad del proceso envuelto en ella. La destilación y reducción de complejidad es ciertamente un aspecto esencial de la representación, "pero típicamente las representaciones añaden tanto como sustraen, y tienen propiedades excedentes que no se corresponden con nada de los fenómenos que describen" (Swoyer 1991, p. 463). Podemos determinar esta otra característica típica de la representación como una inducción de complejidad. Como se mostró en los ejemplos anteriores, el ámbito representante, sea matemático o material, ofrece un conjunto de nuevas posibilidades para tratar con las entidades representadas. $\mathrm{Si}$, en concreto, restringimos nuestra atención al caso de la representación conceptual, el lenguaje del dominio representante contiene muchos conceptos y proposiciones que no pueden traducirse directamente a conceptos y proposiciones del lenguaje que describe el dominio representado. Pero la "nueva" complejidad del dominio representante no es superflua; es esencial para toda representación, en la medida en que se la 
usa para generar nuevo conocimiento acerca del dominio representado. Por ejemplo, en el caso de la medida numérica la rica estructura matemática de los números reales se usa para elaborar una teoría comprehensiva de la aproximación. En general, el auténtico propósito de la representación es la aplicación de la teoría del sistema representante al sistema representado (Mundy 1986, p. 32). De ahí que la invención de una representación apropiada - y de su complejidad inducida - pueda considerarse como el ingrediente esencial de la solución de un problema complicado.

En resumen, una buena representación - que no se reduce a una mera traducción exacta y precisa - es una representación con poder "abductivo", porque procura un razonamiento en el dominio representante que puede transferirse al dominio representado. Es crucial remarcar aquí que tanto la reducción como la inducción de complejidad son características genuinas de la representación que dependen esencialmente del sujeto que interpreta la representación. No hay, pues, "buenas" o "malas" reducciones o inducciones representacionales tout court. La evaluación de las cualidades reductivas e inductivas de una representación depende de los intereses teóricos y/o prácticos del sujeto interpretante.

Haremos mención finalmente a algunas representaciones reflexivas relevantes para la ciencia moderna considerada como parte de la sociedad actual. Las representaciones originales del conocimiento científico no pueden trasladarse sin más a una audiencia más amplia, al conjunto de la sociedad. La divulgación y la popularización son ingredientes necesarios en la comunicación científica. Para bien o para mal, ambas transformaciones representacionales muestran también las características representacionales básicas de la reducción e inducción de complejidad que acaban de mencionarse. Por un lado, se omiten aspectos matemáticos o conceptuales, se criban las distinciones más sutiles, etc. 
Pero por otro lado, otras "re-representaciones" informan el contexto educativo induciendo complejidad. En otras palabras, la ciencia, en tanto que práctica representacional, tiene que ser enseñada y aprendida, y esas actividades incorporan nuevos e importantes nudos a la red representacional de la ciencia ( $c f r$. Echeverría 1995). No hemos tratado aquí ninguno de los asuntos que se mencionaron en este apartado final. Pero ellos conforman una importante agenda que el representacionismo combinatorio no puede dejar de considerar en un futuro próximo.

\section{BIBLIOGRAFÍA}

Cassirer, E., 1910, Substanzbegriff und Funktionsbegriff. Über die Grundlagen der Erkenntniskritik, Darmstadt, Wissenschaftliche Buchgesellschaft, 1980.

Díez, J.A., 1998, "Hacia una teoría general de la representación científica", Theoria 13/1, 113-139.

Duhem, P., 1906, La Théorie Physique, son objet, sa structure, París, Vrin, 1989.

Echeverría, J., 1995, Filosofía de la ciencia, Madrid, Akal.

Galison, P., 1997, Image and Logic. A Material Culture of Microphysics, Chicago, Chicago University Press.

Goldblatt, R., 1979, Topoi - The Categorial Analysis of Logic, Amsterdam, North-Holland.

Gooding, D., 1992, "Putting Agency Back into Experiment", en A. Pickering (ed.), Science as Practice and Culture, Chicago, Chicago University Press, pp. 65-112.

Haack, S., 1993, Evidence and Enquiry. Towards Reconstruction in Epistemology, Oxford, Basil Blackwell.

Hacking, I., 1999, The Social Construction of What?, Cambridge, Mass., Harvard University Press.

Hertz, H., 1894, Die Prinzipien der Mechanik in neuem Zusammenhange dargestellt, 2a. ed. preparada por P. Lenard, Leipzig.

Ibarra, A. y T. Mormann, 1994, "Counterfactual Deformation and Idealization in a Structuralist Framework", en 
M. Kuokkanen (ed.), Idealization VII. Structuralism, Idealization and Approximation (Poznan Studies in the Philosophy of the Sciences and the Humanities, vol. 42), Amsterdam, Rodopi, pp. 81-94.

— , 1998, "Datos, fenómenos, constructos —Un enfoque representacional", Theoria, 13/1, pp. 61-87.

James, W., 1907, Pragmatismo, Barcelona, Orbis, 1985.

Krantz, D.H., Luce, R.D., Suppes, P., Tversky, A., 1971-1990, The Foundations of Measurement, vols. 1, 2 y 3, Nueva York, Academic Press.

Latour, B., 1999, Pandora's Hope. Essays on the Reality of Science Studies, Cambridge, Mass., Harvard University Press. Lawvere, F.W., Schanuel, S.H., 1995, Conceptual Mathematics, Cambridge, Cambridge University Press.

Laymon, R., 1982, "Scientific Realism and the Hierarchical Counterfactual Path form Data to Theory", en P. Asquith, T. Nickles (eds.), PSA 1982, vol. 1, East Lansing, Philosophy of Science Association, 107-121.

— 1987, "Using Scott Domains to Explicate the Notions of Approximate and Idealized Data", Philosophy of Science, 54, 194-221,

Mac Lane, S., 1971, Categories for the Working Mathematician, Berlín, Springer.

Mundy, B., 1986, "On the General Theory of Meaningful Representation", Synthese, 67, 391-437.

Narens, L., 1985, Abstract Measurement Theory, Cambridge, Mass., The MIT Press.

Peirce, C.S., 1973, Lectures on Pragmatism, Hamburgo, Felix Meiner.

Pickering, A., 1995, The Mangle of Practice. Time, Agency, and Science, Chicago, Chicago University Press.

Rorty, R., 1979, Philosophy and the Mirror of Nature, Princeton, Princeton University Press.

Schlick, M., 1918, Allgemeine Erkenntnislehre, Francfort, Suhrkamp, 1979.

Suppes, P., 1989, "Representation Theory and the Analysis of Structure", Philosophia Naturalis, 25, 254-268. 
Swoyer, C., 1991, "Structural Representations and Surrogative Reasoning", Synthese, 87, 449-508.

van Fraassen, B., 1989, Laws and Symmetry, Oxford, Clarendon Press.

Watson, R.A., 1995, Representational Ideas, Dordrecht, Kluwer.

Recibido: 10 de diciembre de 1999 


\section{SUMMARY}

The aim of this paper is to introduce a new concept of scientific representation into philosophy of science. The new concept - to be called homological or functorial representation - is a genuine generalization of the received notion of representation as a structure preserving map as it is used, for example, in the representational theory of measurement. It may be traced back, at least implicitly, to the works of Hertz and Duhem. A modern elaboration may be found in the foundational discipline of mathematical category theory. In contrast to the familiar concepts of representations, functorial representations do not depend on any notion of similarity, neither structural nor objectual one. Rather, functorial representation establish correlations between the structures of the representing and the represented domains. Thus, they may be said to form a class of quite "non-isomorphic" representations. Nevertheless, and this is the central claim of this paper, they are the most common type of representations used in science. In our paper we give some examples from mathematics and empirical science. One of the most interesting features of the new concept is that it leads in a natural way to a combinatorial theory of scientific representations, i.e. homological or functorial representations do not live in insulation, rather, they may be combined and connected in various ways thereby forming a net of interrelated representations. One of the most important tasks of a theory of scientific representations is to describe this realm of combinatorial possibilities in detail. Some first tentative steps towards this endeavour are done in our paper. 